\title{
Erratum to: Clinical and molecular aspects of distal renal tubular acidosis in children
}

\author{
Martine T. P. Besouw ${ }^{1}$ - Marc Bienias ${ }^{2} \cdot$ Patrick Walsh $^{3} \cdot$ Robert Kleta $^{1,5}$. \\ William G. van't Hoff ${ }^{1}$ - Emma Ashton ${ }^{4}$ - Lucy Jenkins ${ }^{4}$ - Detlef Bockenhauer ${ }^{1,5}$
}

Published online: 3 March 2017

(C) IPNA 2017

\section{Erratum to: Pediatr Nephrol}

DOI 10.1007/s00467-016-3573-4

This article contains the following mistakes:

- The mutation in patient 20: c.1181G>A; p.(Arg394Gln) is in the gene ATP6V1B1; this was mislabeled both in the text and in the table

- Three further mutations are novel, but were not highlighted as such:
1. c. $1387 \mathrm{G}>\mathrm{T} ; \mathrm{p} .(\mathrm{Glu} 463 *)$ in $A T P 6 V 1 B 1$ (in patient 1)

2. c. $905 \mathrm{G}>\mathrm{C}$; p. (Arg302Pro) in $A T P 6 V 1 B 1$ (in patient 2$)$

3. c.1107del p. (Asn370Ilefs*22) in ATP6V0A4 (in patient 7)

The authors regret these errors and apologize for any inconvenience caused.

The online version of the original article can be found at http://dx.doi.org/ 10.1007/s00467-016-3573-4.

Detlef Bockenhauer

detlef.bockenhauer@gosh.nhs.uk

1 Department of Pediatric Nephrology, Great Ormond Street Hospital for Children NHS Foundation Trust, Great Ormond Street, London WC1N 3JH, UK

2 Department of Pediatrics, Medical Faculty Carl Gustav Carus, Technical University Dresden, Dresden, Germany

3 Great North Children's Hospital, Newcastle upon Tyne NHS Foundation Trust, Newcastle, UK

4 North East Thames Regional Genetics Service Laboratories, Great Ormond Street Hospital for Children NHS Foundation Trust, London, UK

5 Centre for Nephrology, University College London Institute of Child Health, London, UK 